\title{
Incentives of stock option based compensation
}

\author{
Elettra Agliardi, Rainer Andergassen* \\ Department of Economics, University of Bologna \\ Piazza Scaravilli 2, 40126
}

February 12, 2003

\begin{abstract}
We introduce explicitly the effort as a choice variable in a continuous time utility maximisation framework of an executive who is partly compensated with stock options. We solve the model in the case where the executive is not allowed to trade in the company's stock but is able to achieve a partial insurance through trading in a correlated market portfolio. We define the executive's value of the options through a certainty equivalence approach both in the case of European call options and nonstandard capped stock options and study the behaviour of the reservation price as relevant parameters change.
\end{abstract}

JEL G13, G30, G32, J33, M12

Key words: incentives, executive stock options, unhedgeable risks, utility maximisation, non-standard options compensation.

*E-mail addresses: agliardi@economia.unibo.it (E. Agliardi), anderga@economia.unibo.it (R. Andergassen). 


\section{Introduction}

Executive stock options have become increasingly popular. Murphy (1999) documents that stock option based compensation increased during the last decade at the cost of base salaries in all US sectors, except in the utilities. The debate about executive compensation structures has recently reemerged at the international level, following on the controversial cases such as Enron, Worldcom and others. In principle, the rising importance of stock option compensation for executives is justified by incentive effects: stock option compensation can be highly effective in aligning corporate officers' incentives with those of shareholders. Regrettably, some perverse effects on the quality of corporate disclosures may also emerge whenever such compensations plans do not provide the right incentives. In some cases, executives might adopt strategies to affect firm's evaluation. Moreover, in the current accountings the failure to include the value of most stock options grants as executives compensation and hence, to subtract them from pretax profits, has increased reported earnings and presumably stock prices. In response, an appropriate compensation plan is called for , both to provide the right incentives and to filter out market wide performance so that executives will not be rewarded because of a bull market.

Some have argued that Black-Scholes option pricing, the prevailing means of estimating options, is approximate and is not a good estimate of the value that is transferred to the executive. The Black-Scholes option price is claimed to overstate the value of the options to the executive and consequently it overstates also their incentives (see Lambert et al, 1991; Kulatilaka and Marcus, 1994; Huddart, 1994; Detemple and Sundaresan, 1999; Hall and Murphy, 2000; Henderson 2002). All these papers define the value of the options to the executive based on a certainty equivalence approach. They assume that the executive cannot short sell and therefore cannot hedge exposure: because of such trading restrictions, there will be a wedge in the valuation of the stock option by the executive and the firm, namely, the executive values the option less than the cost to the company.

Johnson and Tian (2000) define incentives of the standard executive stock options as the derivative of the option value (defined by the Black-Scholes formula) with respect to the underlying asset price, i.e. the Black-Scholes delta. Thus, incentives are larger and the executive's effort is larger, the larger is delta. But since the Black-Scholes value overstates the executive value, also the incentives based on this computation will overstate the true incentives.

Hall and Murphy (2002) and Henderson (2002) consider incentives as the derivative of the utility based evaluation of the options with respect to the asset price: in this way, they try to capture the executive's incentive to influence the firm's stock price through his actions.

In this paper we tackle again the issue of the value and incentive effects of stock option compensation. We extend the continuous time utility maximisation model proposed by Henderson (2002) by introducing the effort of executives explicitly. Akin to the static model proposed by Jenter (2001) we explicitly introduce effort as a choice variable for the executive. In particular, we will 
assume that the executive is able to increase the long run drift of the asset price through an increase in the effort. Thus, the larger is the effort, the larger will be the drift of the asset price and consequently the larger is the value of the options. On the other hand, a larger effort reduces the utility of the executive directly. Thus, the executive solves this trade-off by choosing the optimal amount of effort in each period of time.

We calculate explicitly the optimal effort and the option value for the executive for two types of compensation plans: the case of a standard European call option and the case of a non-standard capped stock option, which has the property that the recipient can benefit from the increase of the underlying asset price only up to a predetermined barrier. This latter plan seems indeed to be more appropriate to filter out market wide performance. Moreover, we study the behaviour of the reservation price as relevant parameters change for both cases and find sufficient conditions such that non-standard capped stock options are more appealing than standard call options.

The remaining part of this paper is organised as follows. In Section 2 we outline the basics of the model and find the reservation price for the two contingent claims. In Section 3 we study the case of standard European call options, while in Sections 4 we study the case of non-standard capped stock options. Section 5 concludes.

\section{The model}

We consider an executive receiving a claim on the company's stock $S$ on which trading is not allowed. The executive can take positions in the market portfolio $M$, the dynamics of which are assumed to follow:

$$
\frac{d M}{M}=\mu d t+\sigma d B
$$

where $B$ is a Wiener process. Under appropriate measure, it can be written as

$$
\frac{d M}{M}=r d t+\sigma d B^{o}
$$

where $B^{o}$ is a Wiener process, $B^{o}=B+\frac{\mu-r}{\sigma} t$ and $r$ is the riskless rate of return. We assume that the dynamics of the company's stock $S$ are given by the following

$$
\frac{d S}{S}=(\nu+\alpha f) d t+\eta\left(\rho d B+\sqrt{1-\rho^{2}} d W\right)
$$

where $W$ is a Wiener process independent of $B, f$ is the executive's effort and $\alpha$ indicates the ability of the executive to influence the drift of the company's stock. Further, we assume that the effort level is not observable by the principal.

The risk component of the stock can be split into the market or systematic risk $\eta^{2} \rho^{2}$ and the firm-specific or non-systematic risk $\eta^{2}\left(1-\rho^{2}\right)$. Thus, for $\rho=0$ all the risk is firm-specific, while for $|\rho|=1$ all the risk is market specific. Since the executive is not allowed to trade in the company's stock he cannot 
construct a riskless portfolio. Thus, if $\rho=1$ the risk can be diversified away, while if $\rho=0$ the risk cannot be hedged away. As long as $|\rho|<1$ the executive is able to reach a partial insurance by market trading.

The executive faces a portfolio allocation problem. In particular, he has to determine the amount of wealth he wants to invest in the market portfolio and the amount of wealth he wants to invest in the riskless asset. Let $\theta$ denote the amount of wealth $X$ to be invested in the market portfolio; consequently, $X-\theta$ denotes the amount the executive wants to invest in the riskless asset yielding an interest rate $r$. Thus,

$$
d X=\theta \frac{d M}{M}+r(X-\theta) d t
$$

and after rearranging terms

$$
d X=\theta \sigma d B+\theta(\mu-r) d t+r X d t
$$

The executive receives $\lambda \geq 0$ units of a stock option whose payoff is $h\left(S_{T}\right)$. In what follows we will consider two special cases: a) a standard European call option, i.e. $h\left(S_{T}\right)=\left(S_{T}-K\right)^{+}$and b) a non-standard capped stock option (see Agliardi, 2002), i.e. $h\left(S_{T}\right)=\operatorname{Min}\left[\left(S_{T}-K\right)^{+}, \bar{S}-K\right]$, where $\bar{S}>K$ is fixed. In the case of capped options we have that $0 \leqslant h\left(S_{T}\right) \leqslant \bar{S}-K$, i.e. the payoff is bounded, while in the case of call options $h\left(S_{T}\right) \geqslant 0$, i.e. the payoff is unbounded from above.

We assume that the effort exerted by the executives reduces his utility. Thus, effort represents a disutility. In particular, we assume that the disutility of effort is increasing in effort and convex. In order to keep the model analytically tractable, we also assume that the utility function is additively separable in wealth and effort.

The executive has two choice variables: the optimal amount of wealth invested in the market portfolio and the optimal level of effort. His value function is given by

$$
V\left(t, X_{t}, S_{t} ; \lambda\right)=\sup _{\left(\theta_{u}, f_{u}\right)_{u \geqslant 0}} E_{t}\left(U\left(X_{T}-\beta \frac{f_{t}^{2}}{2} t+\lambda h\left(S_{T}\right)\right)\right)
$$

Using the assumption of CARA utility function, i.e. $U(x)=-\frac{1}{\gamma} e^{-\gamma x}, \gamma>0$ we can rewrite (1) as follows

$$
V\left(t, X_{t}, S_{t} ; \lambda\right)=-\frac{1}{\gamma} e^{-\gamma\left(e^{r(T-t)} X_{t}-\beta \frac{f_{t}^{2}}{2} t\right)} g\left(T-t, \log S_{t}\right)
$$

where $g\left(0, \log S_{T}\right)=e^{\lambda \gamma h\left(S_{T}\right)}$.

The following restrictions on the values of the parameters $\alpha$ and $\beta$ are required in order to obtain an admissible solution in the case of standard European call options. 
Assumption 1 Let $\frac{\alpha^{2}}{\beta}<\left(1-\rho^{2}\right) \gamma \eta^{2}$ for $h\left(S_{T}\right)=\left(S_{T}-K\right)^{+}$

In Proposition 1 we state the solution for the optimal strategy $\left(\theta_{t}^{*}, f_{t}^{*}\right)$.

Proposition 1 Let Assumption 1 hold. The optimal strategy $\left(\theta_{t}^{*}, f_{t}^{*}\right)$ is given by

$$
\begin{aligned}
\theta_{t}^{*} & =\frac{(\mu-r) g+g_{S} \sigma \eta \rho}{\gamma e^{r(T-t)} g \sigma^{2}} \\
f_{t}^{*} & =-\frac{\alpha g_{S}}{\beta \gamma g}
\end{aligned}
$$

where $g\left(T-t, \log S_{t}\right)=e^{-\frac{(\mu-r)^{2}}{2 \sigma^{2}}(T-t)}\left[E^{0}\left(e^{-\frac{\gamma \lambda}{b} h\left(S_{t} e^{\Omega_{t}}\right)}\right)\right]^{b}, b=\frac{1}{1-\rho^{2}-\frac{\alpha^{2}}{\gamma \beta \eta^{2}}}$, $\Omega_{t}=\left(\nu-\frac{\eta \rho}{\sigma}(\mu-r)-\frac{1}{2} \eta^{2}\right)(T-t)+\eta z_{t}$ and $z_{t}$ is a Brownian motion.

Proof. Applying Ito to (2) we have that the deterministic terms are

$$
\begin{gathered}
-e^{r(T-t)} \gamma \theta(\mu-r) g+\frac{1}{2} \gamma^{2}\left(e^{r(T-t)}\right)^{2} \theta^{2} \sigma^{2} g-\gamma e^{r(T-t)} \theta \sigma \rho \eta g_{S}+ \\
+\gamma \frac{f^{2}}{2} g \beta-g_{t}+g_{S}\left(\nu+\alpha f-\frac{1}{2} \eta^{2}\right)+\frac{1}{2} g_{S S} \eta^{2}=0
\end{gathered}
$$

Maximising (4) over $\theta$ and $f$ we obtain (3). Substituting (3) back into (4) and rearranging terms we obtain the following pde:

$$
\begin{aligned}
&-g_{t}+g_{S}\left(\nu-\frac{1}{2} \eta^{2}\right)+\frac{1}{2} g_{S S} \eta^{2}-\alpha^{2} \frac{g_{S}^{2}}{2 g \beta \gamma}+ \\
&-\frac{\left[(\mu-r) g+g_{S} \sigma \eta \rho\right]^{2}}{2 g \sigma^{2}}=0
\end{aligned}
$$

Following Zariphopoulou (2001) and Henderson and Hobson (2002) we make the substitution $g(\tau, y)=e^{\delta \tau} G(\tau, y+\phi \tau)^{b}$. Using $\delta=-\frac{(\mu-r)^{2}}{2 \sigma^{2}}, \phi=\nu-\eta \frac{\rho(\mu-r)}{\sigma}-$ $\frac{1}{2} \eta^{2}, \tau=T-t$ and $b=\frac{1}{1-\rho^{2}-\frac{\alpha^{2}}{\gamma \beta \eta^{2}}}(5)$ reduces to the pde

$$
G_{\tau}=\frac{1}{2} \eta^{2} G_{S S}
$$

subject to $G(0, y)=e^{-\frac{\gamma \lambda}{b} h\left(e^{y}\right)}$.

The fundamental solution to $(6)$ is

$$
w(\tau, y)=\frac{1}{\sqrt{2 \pi \eta^{2} \tau}} e^{-\frac{y^{2}}{2 \eta^{2} \tau}}
$$

where $\int_{-\infty}^{+\infty} w(\tau, y) d y=1$. Thus,

$$
G(\tau, y)=E(G(0, y+\eta z))
$$

where $z$ is a Brownian motion. Substituting this last expression back into $g(\tau, y)$ we obtain the result stated in Proposition 1.

Notice that the structure of $f^{*}$ is very similar to the incentives derived in Henderson (2002). For small values of $\lambda$ we observe that the incentives are 
increasing in $\frac{\alpha}{\beta}$ and vanish to the first order as $\lambda$.tends to 0 . Thus, for $\lambda=0$ we have that incentives are zero. The intuition for this result is that if the executive does not receive any contigent claim, then it is optimal not to exert any effort since effort represents a disutility. Observe that $\partial f^{*} / \partial \eta<0$, that is, incentives decrease with volatility. Moreover, $\partial f^{*} / \partial \rho<0$.

Given an initial wealth $\mathrm{x}$, the reservation price $p$ is defined implicitly as

$$
V(0, x-p, S ; \lambda)=V(0, x, S ; 0)
$$

Straightforward computation allows us to get Proposition 2:

Proposition 2 The reservation price $p$ is given by

$$
p_{t}=-\frac{b}{\gamma} e^{-r(T-t)} \log E^{0}\left[e^{-\frac{\lambda \gamma}{b} h\left(S_{t} e^{\Omega_{t}}\right)}\right]-\frac{1}{2} \beta e^{-r(T-t)} f_{t}^{2} t
$$

where $f_{t}$ can be computed as from (3).

In the next sections we will make use of the following assumption.

$$
\text { Assumption } 2 \alpha=\alpha^{\prime} \sqrt{1-\rho^{2}}
$$

Assumption 2 states that the executive's ability to influence the long run drift of the company's asset depends on $\rho$. In particular, if $\rho=0$, i.e. all the risk is firm specific, the executive is most efficient in influencing the dynamics of the asset. Thus, $\rho$ indicates the efficiency of the action of the executive in influencing the dynamics of the asset.

\section{Standard European Call options}

The cost of each option for the firm is given by the Black-Scholes formula $c(S, t)=e^{-r(T-t)} E^{0}\left(S_{T}-K\right)^{+}$, while the value the executive attaches to a single option is given by $\frac{p}{\lambda}$, where $p$ can be computed by $(7)$ with $h\left(S_{t} e^{\Omega t}\right)=$ $\left(S_{t} e^{\Omega t}-K\right)^{+}$. The difference between the cost of the option and the value the executive attaches to the single option is known as the deadweight cost and is defined by $c(S, t)-\frac{p}{\lambda}$.

In order to study the behaviour of the reservation price, we take a second order approximation for low values of $\lambda$ :

$$
\begin{aligned}
\frac{p_{t}}{\lambda} \approx & e^{-r(T-t)}\left\{E^{0}\left(S_{t} e^{\Omega_{t}}-K\right)^{+}-\frac{\lambda}{2}\left(1-\rho^{2}\right) \frac{\alpha^{\prime 2}}{\beta} \times\right. \\
& \left.\times\left[\gamma\left(\frac{\beta}{\alpha^{\prime 2}}-\frac{1}{\gamma \eta^{2}}\right) E^{0}\left[\left(S_{t} e^{\Omega_{t}}-K\right)^{+}\right]^{2}+t\right]\right\}
\end{aligned}
$$

We are able to recover in this case the result stated in Henderson (2002) that the reservation price converges towards the cost of the option if $\rho \rightarrow 1$. This result is stated formally in the following Corollary. 


\section{Corollary 1}

1. $\frac{p}{\lambda} \underset{\rho \rightarrow 1}{\longrightarrow} c(S, t)$

2. $f_{t}^{*} \underset{\rho \rightarrow 1}{\longrightarrow} 0$

Corollary 1 states that if all the risk becomes insurable, then the reservation price per unit of option converges towards the cost of each option. Thus, as $\rho \rightarrow 1$, the deadweight cost converges towards zero. This is a direct consequence of the result that the effort $f_{t}^{*}$ converges towards zero as all the risk becomes insurable.

Part 2) of Corollary 1 states that as $\rho \rightarrow 1$, the level of effort $f_{t}^{*}$ converges towards zero. This result is not because of vanishing incentives, but because of the vanishing ability of influencing the dynamics of the company's asset, i.e. $\alpha \rightarrow 0$.

We are interested in the behaviour of the reservation price as $\rho$ changes:

$$
\begin{gathered}
\frac{\partial}{\partial \rho}\left(\frac{p_{t}}{\lambda}\right) \approx e^{-r(T-t)}\left\{\frac{\partial}{\partial \rho} E^{0}\left(S_{t} e^{\Omega_{t}}-K\right)^{+}+\right. \\
+\lambda \rho \frac{\alpha^{\prime 2}}{\beta}\left[\gamma\left(\frac{\beta}{\alpha^{\prime 2}}-\frac{1}{\gamma \eta^{2}}\right) E^{0}\left[\left(S_{t} e^{\Omega_{t}}-K\right)^{+}\right]^{2}+t\right]+ \\
-\frac{\lambda}{2} \gamma\left(1-\rho^{2}\right)\left(1-\frac{\alpha^{\prime 2}}{\beta \gamma \eta^{2}}\right) \frac{\partial}{\partial \rho} E^{0}\left[\left(S_{t} e^{\Omega_{t}}-K\right)^{+}\right]^{2}
\end{gathered}
$$

We observe that the first term of (9) is negative, while the second and third terms are positive. If the first term dominates the other effects, then the reservation price is decreasing in $\rho$ and consequently $\frac{p_{t}}{\lambda} \geqslant c(S, t)$. In particular, for sufficiently small values of $\lambda$ we have that $\frac{p_{t}}{\lambda} \geqslant c(S, t)$.

Consider the effect of a change in the volatility $\eta^{2}$ on the reservation price $\frac{p_{t}}{\lambda}$. Taking the first derivative of the reservation price with respect to $\eta^{2}$ we obtain

$$
\begin{gathered}
\frac{\partial}{\partial \eta^{2}}\left(\frac{p_{t}}{\lambda}\right) \approx e^{-r(T-t)}\left\{\frac{\partial}{\partial \eta^{2}} E^{0}\left(S_{t} e^{\Omega_{t}}-K\right)^{+}+\right. \\
-\frac{\lambda}{2}\left(1-\rho^{2}\right) \frac{\alpha^{\prime 2}}{\beta}\left(\frac{1}{\eta^{2}}\right)^{2} E^{0}\left[\left(S_{t} e^{\Omega_{t}}-K\right)^{+}\right]^{2}+ \\
-\lambda \gamma\left(1-\rho^{2}\right)\left(1-\frac{\alpha^{\prime 2}}{\beta} \frac{1}{\gamma \eta^{2}}\right) \frac{\partial}{\partial \eta^{2}} E^{0}\left[\left(S_{t} e^{\Omega_{t}}-K\right)^{+}\right]^{2}
\end{gathered}
$$

We observe that the first term and the second term of (10) are negative, while the third term is positive. If the first two terms dominate the third one, then the reservation price is decreasing in $\eta^{2}$. In particular, for $\lambda$ sufficiently small we have that $\frac{\partial}{\partial \eta^{2}}\left(\frac{p_{t}}{\lambda}\right) \leqslant 0$, that is, the executive prefers less volatile environments.

These results are summarised in the following Corollary

Corollary 2 For $\lambda$ sufficiently small we have that:

a) $\frac{\partial}{\partial \rho}\left(\frac{p_{t}}{\lambda}\right) \leqslant 0$

b) $\frac{p_{t}}{\lambda} \geqslant c(S, t)$

c) $\frac{\partial}{\partial \eta^{2}}\left(\frac{p_{t}}{\lambda}\right) \leqslant 0$ 
Finally, we get $\frac{\partial}{\partial \gamma}\left(\frac{p_{t}}{\lambda}\right) \leqslant 0$, that is, higher risk aversion let the executive place a lower value on options.

\section{Non-standard capped stock options}

The payoff structure of a capped option is, by definition, bounded. Observe that as long as $b>0$ the payoff structure remains concave, while for $b<0$ we have that the payoff structure becomes convex. We will see that this has important implications.

From Agliardi (2002) we know that the cost of the capped stock option for the firm $\hat{c}(S, t)$ is lower than the cost of a standard call option, i.e. $\hat{c}(S, t)<c(S, t)$. This result is because of the limitation in potential returns.

Taking a second order approximation of the reservation price for small values of $\lambda$ we get

$$
\begin{aligned}
\frac{\hat{p}_{t}}{\lambda} & \approx e^{-r(T-t)}\left\{E^{0} \operatorname{Min}\left(S_{t} e^{\Omega_{t}}-K\right)^{+}, \bar{S}-K\right]-\frac{\lambda}{2}\left(1-\rho^{2}\right) \frac{\alpha^{\prime 2}}{\beta} \times \\
& \left.\times\left[\gamma\left(\frac{\beta}{\alpha^{\prime 2}}-\frac{1}{\gamma \eta^{2}}\right) E^{0}\left[\operatorname{Min}\left[\left(S_{t} e^{\Omega_{t}}-K\right)^{+}, \bar{S}-K\right]\right]^{2}+t\right]\right\}
\end{aligned}
$$

We can state the following Corollary:

\section{Corollary 3}

1. $\frac{\hat{p}}{\lambda} \underset{\rho \rightarrow 1}{\longrightarrow} \hat{c}(S, t)$

2. $f_{t}^{*} \underset{\rho \rightarrow 1}{\longrightarrow} 0$

Corollary 3 states that if all the risk becomes insurable, then the reservation price per unit of option converges towards the cost of each option. Thus, as $\rho \rightarrow 1$, the deadweight cost converges towards zero. Furthermore, incentives and the effort become vanishing small as $\rho$ increases towards 1 .

\section{Corollary 4}

A sufficient condition for $\frac{\hat{p}}{\lambda} \geq \hat{c}(S, t)$ to be true is either

1. $\gamma\left(\frac{1}{\gamma \eta^{2}}-\frac{\beta}{\alpha^{\prime 2}}\right) E^{0}\left[\operatorname{Min}\left[\left(S_{t} e^{\Omega_{t}}-K\right)^{+}, \bar{S}-K\right]\right]^{2}>t$, or

2. $\lambda$ sufficiently small.

Observe that at time $t=0$, Corollary 4, Part 1), is always satisfied, if $b<0$, i.e. $\alpha^{\prime 2}>\gamma \beta \eta^{2}$, and consequently at the initial time the executive values the capped options more than they cost the firm. Condition $\alpha^{2}>\gamma \beta \eta^{2}$ states that the executives ability to influence the drift of the company's asset 
is sufficiently large. Thus, at the initial time the company finds capped options more attractive than call options since the former lead to a negative deadweight loss.

Comparing capped options with standard call options, the possibility that $b<0$ in the case of capped options increases the probability that the reservation price is larger than the cost of the option.

To summarize the comparison between capped stock options and standard call options we observe that the former are much cheaper than the latter and further, the probability of observing a negative deadweight cost is larger in the case of capped options than in the case of call options.

In order to make a comparison between the two compensations we provide numerical simulations, in the case where we vary the number of options granted by the firm such that the total cost of the two portfolios is the same. In particular, we fix the number of call options granted $\lambda$ and consequently the number of capped stock options is $\frac{\lambda c(S, t)}{\hat{c}(S, t)}$. In Figure 1 and 2 we provide numerical simulations of the value the executive attaches at time $t=0$ to call options (dashed line) and to capped stock options (continuous line) as a function of $\rho$. The value of the parameters we used in the simulations are: $r=0.05, S_{0}=K=100$, $\mu=0.1, \tau=8, \sigma=0.35, \gamma=\frac{4}{10^{7}}, \lambda=25.000$ and $1-\frac{\alpha^{\prime 2}}{\beta \gamma \eta^{2}}=0.5$.
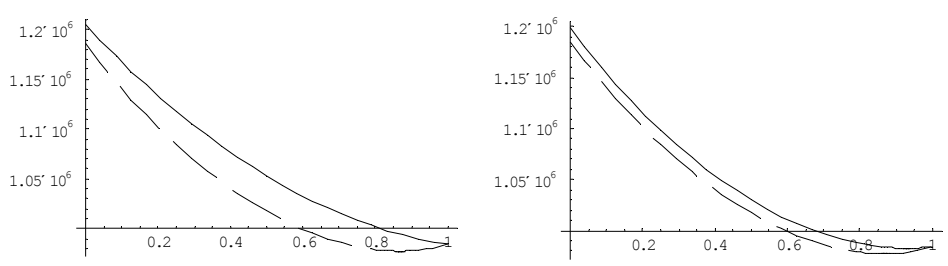

Figure 1: $\eta=0.2$; LHS $\bar{S}=220$ and RHS $\bar{S}=420$.
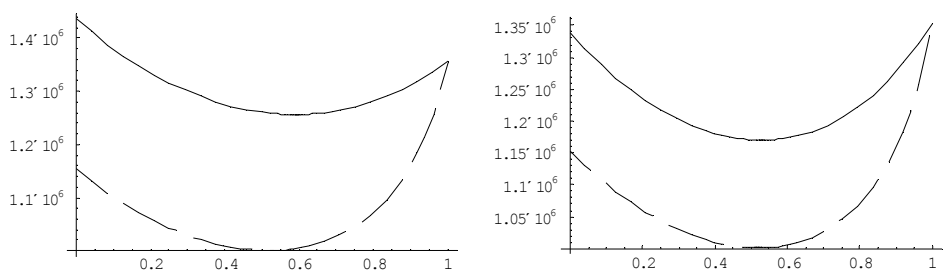

Figure $2: \eta=0.4 ;$ LHS $\bar{S}=220$ and RHS $\bar{S}=420$.

From Figures 1 and 2 we observe that capped stock options lead to a larger reservation price than standard European call options. 


\section{Conclusion}

We solved the continuous time maximisation problem of an executive who is partially compensated with either European call options or non-standard capped stock option. Optimal effort, optimal portfolio and the option value for the executive are derived in the case where he is not allowed to trade in the company's stock but where he can achieve partial insurance through trading in a correlated market portfolio. For both types of options we studied the behaviour of the reservation price as relevant parameters change and showed conditions under which the executive values the options more than they cost the firm.

\section{References}

[1] Agliardi E., 2002, "Pip vs Groi Portfolios", mimeo.

[2] Detemple J. and S. Sundaresan, 1999, "Nontraded Asset Valuation with Portfolio Constraints: A Binomial Approach", Review of Financial Studies, $12(4), 835-872$.

[3] Hall B.J. and K.J. Murphy, 2000, "Optimal Exercise Prices for Executive Stock Options", American Economic Review, 90 (2), 209 - 214.

[4] Henderson V., 2002, "Stock Based Compensation: Firm-specific risk, Efficiency and Incentives", Oxford Financial Research Centre Preprint 2002FE-01.

[5] Henderson V. and D. Hobson, 2002, "Real Options with Constant Relative Risk Aversion", Journal of Economic Dynamics and Control, 27 (2), 329 355 .

[6] Huddart S., 1994, "Employee Stock Options", Journal of Accounting and Economics, 18, 207 - 231.

[7] Jenter D, 2001, "Understanding High-Powered Incentives", Working paper, Harvard Business School and Economics Department.

[8] Johnson S.A. and Y.S. Tian, 2000, "The Value and Incentive Effects of Nontraditional Executive Stock Option Plans", Journal of Financial Economics, 57, 3 - 34 .

[9] Kulatilaka N. and A.J. Marcus, 1994, "Valuing Employee Stock Options", Financial Analysts Journal, 50 (6), 46 - 56.

[10] Lambert R.A., K.F. Larcker and R.E. Verrecchia, 1991, "Portfolio Considerations in Valuing Executive Compensation", Journal of Accounting Research, 29, (1), 129 - 149.

[11] Murphy K., 1999, "Executive Compensation", in Orley Ashenfelter and David Card (eds.), Handbook of Labor Economics, Vol. 3, North Holland. 
[12] Zariphopoulou T., 2001, "A Solution Approach to Valuation with Unhedgeable Risks", Finance Stochastics V (1), 61 - 82. 